\title{
DEVELOPMENT AND EVALUATION OF A PHANTOM FOR MULTI-PURPOSE DOSIMETRY IN INTENSITY-MODULATED RADIATION THERAPY
}

\author{
HAE SUN JEONG ${ }^{1,2}$, YOUNGYIH HAN ${ }^{* 3}$, OYEON KUM ${ }^{4}$, CHAN HYEONG KIM ${ }^{1}$, and JOO HWAN PARK ${ }^{2}$ \\ ${ }^{1}$ Department of Nuclear Engineering, Hanyang University \\ 17 Haengdang-dong, Seongdong-gu, Seoul 133-791, South Korea \\ ${ }^{2}$ Korea Atomic Energy Research Institute \\ 1045 Daedoek-daero, Yuseong-gu, Daejeon 305-353, South Korea \\ ${ }^{3}$ Department of Radiation Oncology, Samsung Medical Center, Sungkyunkwan University School of Medicine \\ 50 Irwon-dong, Gangnam-gu, Seoul 135-710, South Korea \\ ${ }^{4}$ Department of Radiation Oncology, Yonsei University College of Medicine \\ 250 Seongsanno Seodaemun-gu, Seoul 120-752, South Korea \\ *Corresponding author. E-mail : youngyih@skku.edu
}

Received October 02, 2010

Accepted for Publication April 02, 2011

A LEGO-type multi-purpose dosimetry phantom was developed for intensity-modulated radiation therapy (IMRT), which requires various types of challenging dosimetry. Polystyrene, polyethylene, polytetrafluoroethylene (PTFE), and polyurethane foam (PU-F) were selected to represent muscle, fat, bone, and lung tissue, respectively, after considering the relevant mass densities, elemental compositions, effective atomic numbers, and photon interaction coefficients. The phantom, which is composed of numerous small pieces that are similar to LEGO blocks, provides dose and dose distribution measurements in homogeneous and heterogeneous media. The phantom includes dosimeter holders for several types of dosimeters that are frequently used in IMRT dosimetry. An ion chamber and a diode detector were used to test dosimetry in heterogeneous media under radiation fields of various sizes. The data that were measured using these dosimeters were in disagreement when the field sizes were smaller than $1.5 \times 1.5 \mathrm{~cm}^{2}$ for polystyrene and PTFE, or smaller than $3 \times 3 \mathrm{~cm}^{2}$ for an air cavity. The discrepancy was as large as $41 \%$ for the air cavity when the field size was $0.7 \times 0.7 \mathrm{~cm}^{2}$, highlighting one of the challenges of IMRT small field dosimetry. The LEGO-type phantom is also very useful for two-dimensional dosimetry analysis, which elucidates the electronic dis-equilibrium phenomena on or near the heterogeneity boundaries.

KEYWORDS : IMRT, Phantom, Dosimetry, Heterogeneity

\section{INTRODUCTION}

Intensity-modulated radiation therapy (IMRT), an advanced modality of high-precision radiotherapy, is widely used in cancer clinics. The primary advantage of IMRT is that the radiation dose conforms precisely to the three-dimensional volume of a tumor. Because the dose is aimed precisely at the tumor volume, the dose can be increased without increasing the risk to nearby critical organs. In order to achieve such high conformity, radiation fluence often has complex shapes, and dose distributions exhibit sharp gradients at their boundaries [1-4]. Thus, in order to successfully carry out this state-of-the-art treatment, an intensive dosimetry is necessary during the implementation of the technique and treatmentverification processes [5].
IMRT dosimetry can be performed efficiently when a well-designed IMRT-dedicated phantom is available. The required characteristics of the phantom can be summarized as follows:

First, the phantom needs to be able to provide dosimetry not only in homogeneous tissue-equivalent media, but also in heterogeneous environments that physically resemble lungs or bones. This characteristic is particularly important for IMRT commissioning, since disequilibrium at the boundary of different tissues is known to be more pronounced in small radiation fields [6-7]. The accuracy of doses computed by radiotherapy treatment planning systems, therefore, needs to be verified [8].

Second, the design of the phantom needs to allow for dose measurements with diverse types of radiation detectors, because choosing a suitable detector for small 
radiation fields in IMRT can be a challenging and even confounding task [9-11]. In addition, the positions of the heterogeneous structures and the detectors in the phantom need to be geometrically adjustable to accommodate an analysis of dose and dose distributions under various conditions.

To the best of our knowledge, however, none of the commercially available phantoms simultaneously satisfy all of the aforementioned requirements. Rather, commercial phantoms entail restrictions in measuring the doses and the dose distributions that are specific to individual patient treatment conditions [12-14]. In the present study, for IMRT commissioning and treatment plan validation, a diversely configurable LEGO-type phantom was designed and manufactured in order to increase the efficiency of IMRT dosimetry under various practical conditions.

\section{MATERIALS AND METHOD}

\subsection{LEGO-type Multi-purpose Dosimetry Phantom}

The developed phantom is to be used to simulate a volume of body tissue in its interaction with ionizing radiation; therefore, selecting the phantom material is an important issue. In this study, phantom materials were chosen for four human body tissues: muscle, fat, bone, and lung. In order to select phantom materials that are physically similar to human body tissues, several physical properties were considered, including mass density, elemental composition, effective atomic number, mass attenuation coefficient, and mass energy absorption coefficient [15].

The effective atomic number was calculated using the following formula [16]:

$$
Z_{\text {eff }}=A_{\text {eff }}\left[\sum w_{\mathrm{i}} \frac{Z_{\mathrm{i}}}{A_{\mathrm{i}}}\right],
$$

where $w_{\mathrm{i}}, Z_{\mathrm{i}}$, and $A_{\mathrm{i}}$ are the atomic weight fraction, the atomic number, and the atomic mass number corresponding to element ' $\mathrm{i}$ ', respectively, and $A_{\text {eff }}$ is the effective atomic mass number $\left(A_{\text {eff }}=\sum_{i} w_{i} A_{i}\right)$. The mass attenuation coefficients and the mass energy absorption coefficients [17] were taken from a web-based database of the National Institute of Standards and Technology (NIST).

The basic design of the multi-purpose plastic phantom was based on a LEGO-type structure. Figure 1 shows the conceptual configuration of the phantom, which was designed to be transversely and vertically sectioned into multi-size slabs for different dosimetric applications. Thus, the phantom can be altered for different dosimetric conditions, though the standard shape is a $20 \times 20 \times 20 \mathrm{~cm}^{3}$ cube. Heterogeneous materials and radiation detectors can easily be inserted into any slab of the phantom. For absolute and relative dosimetry, the phantom provides dosimeter holders for a variety of dosimeters, including the following: a pinpoint ion chamber (type 31015, PTWFeiburg, Germany), a diode detector (SFD, Scanditronix Wellhofer, Germany), a Markus chamber (type 23343, PTW-Feiburg, Germany), a Roos chamber (type 34001, PTW-Feiburg, Germany), a MOSFET (model TN-1002RD, Thomson and Nielsen Electronics Ltd., Canada), and various radiographic/radiochromic films. The pinpointtype ion chamber has a small sensitive volume and superior spatial resolution compared with other ion chambers. The SFD diode detector is very robust so as to be independent of bias, pressure, and moisture, and it requires no "warmup" time. The Markus and Roos chambers are planeparallel type chambers with vented sensitive volumes of $0.055 \mathrm{~cm}^{3}$ and $0.35 \mathrm{~cm}^{3}$, respectively. Finally, due to its small size, a MOSFET has properties of a 360 degree isotropic response and little beam attenuation.

\subsection{Point-dose Measurement in Small Radiation Fields}

The developed phantom was tested by measuring doses under both homogeneous and heterogeneous conditions, as shown in Figure 2. For the heterogeneous condition, $3 \times 3 \times 3 \mathrm{~cm}^{3}$ of heterogeneous materials (bone-substitute material and air cavity) were individually inserted into the center of the phantom at a depth of $10 \mathrm{~cm}$ from the surface. Among the diverse dosimeters able to be used with the phantom, two were selected to be employed

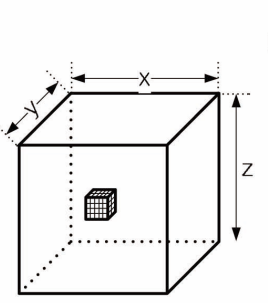

( a )

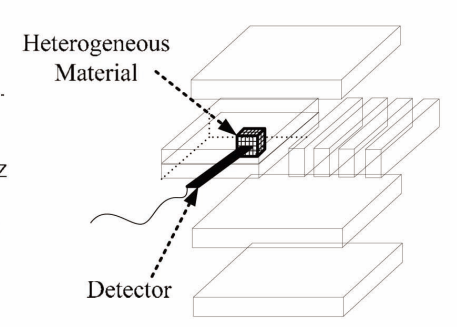

(b)

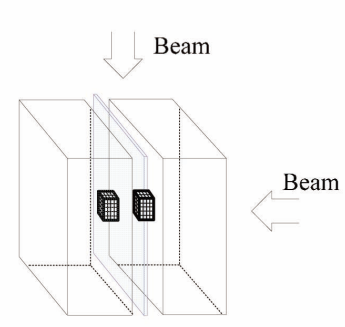

(c)

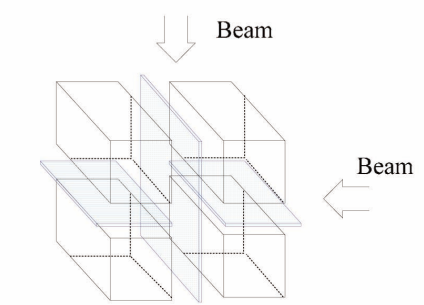

(d)
Fig. 1. Conceptual Configuration of Multi-purpose Dosimetry Phantom: (a) Basic Phantom Design Including Heterogeneous Material, (b) Phantom Slabs Containing Holes for Diverse Heterogeneous Materials and Detectors, the Heterogeneity Position can be Changed. (c) Film Sandwiched between Heterogeneous Materials, and (d) Simultaneous Measurement of Vertical and Lateral Dose Distributions 
for the dose measurements: an ion chamber with a sensitive volume of $0.03 \mathrm{~cm}^{3}$ and a diode detector with an active volume width of $0.006 \mathrm{~cm}$, both of which are commonly used in IMRT QA. By using 6 MV photon beams delivered from a linear accelerator (6EX Clinac, Varian Medical Systems Inc, USA), the output factors at a source-axis distance (SAD) of $100 \mathrm{~cm}$ were measured as a function of the field sizes ranging from $5 \times 5 \mathrm{~cm}^{2}$ to $0.5 \times 0.5 \mathrm{~cm}^{2}$.

\subsection{Dose Distribution Measurement in Phantom}

The dose distributions were also measured using films under homogeneous and heterogeneous conditions with an air cavity and a high-density structure (bone-substitute material) at the center (see Figure 3). GAFCHROMIC ${ }^{\circledR}$ EBT film (batch-number 47277-03I, International Specialty Products, Wayne, NJ) was sandwiched between the phantom slabs parallel to the beam axis through the

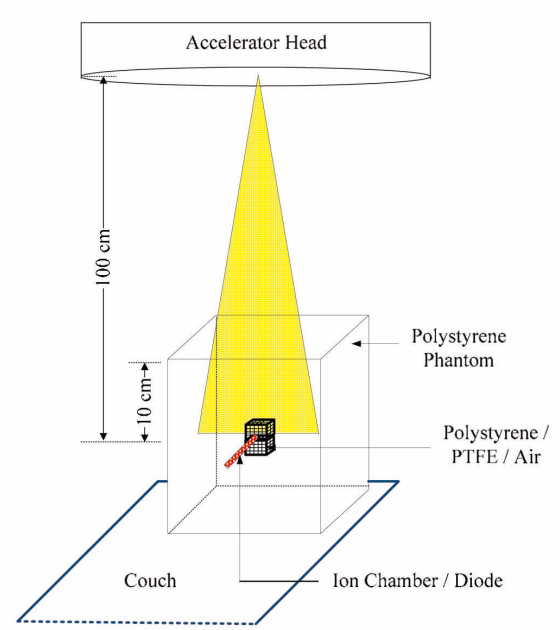

Fig. 2. Experimental Configuration for Point Dose Measurements

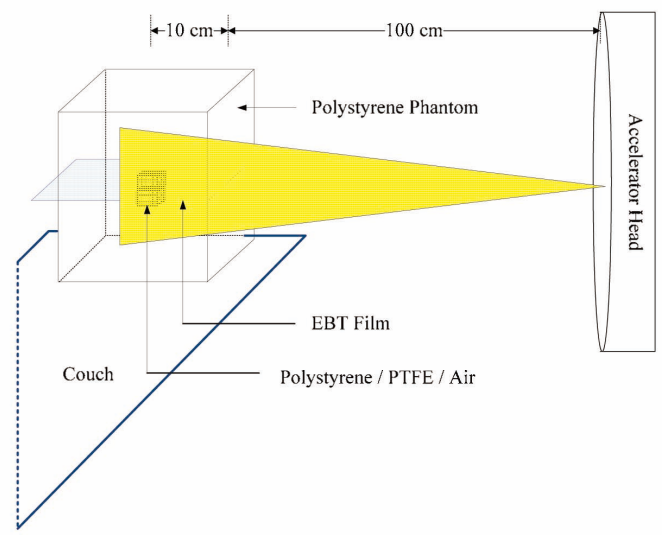

Fig. 3. Experimental Configuration for Percent Depth Dose Measurements heterogeneous materials, as shown in Figure 1 (c). The percentage depth dose (PDD) on the film was read using an Epson 1680 Pro flatbed scanner (Epson Seiko Corporation, Nagano, Japan).

\section{RESULTS AND DISCUSSION}

\subsection{LEG0-type Multi-purpose Dosimetry Phantom}

Many different materials were analyzed by comparing their physical properties with those of the body tissues. Finally, polystyrene, polyethylene, polytetrafluoroethylene (PTFE), and polyurethane foam (PU-F) were selected as the alternatives to muscle, fat, bone, and lung tissue, respectively. Table 1 shows the mass densities and effective atomic numbers of the selected materials. First, the densities of the selected materials were in good agreement with the body tissues (difference: $<4 \%$ ). The effective atomic numbers of the selected materials were also similar to those of the tissues, except for bone (H: $3.4 \%$, C: $15.5 \%$, $\mathrm{N}: 4.2 \%$, O: 43.5\%, Na: 0.1\%, Ma: 0.2\%, P: $10.3 \%$, S: $0.3 \%$, Ca: $22.5 \%$ ) and PTFE (C: $22 \%, \mathrm{~N}: 2 \%, \mathrm{O}: 28 \%, \mathrm{~F}$ : $48 \%)$. The high atomic number of bone's calcium component causes an increase in the interaction coefficients for low energy photons. Nevertheless, PTFE was selected as the best surrogate for bone because the mass attenuation coefficient data agree well for 0.1 to $10 \mathrm{MeV}$ of photons, which is the principal energy range of IMRT X-rays (see Figure 4). Note that $95 \%$ of photons in clinically used $X$ ray beams have energy within the range of $0.1-10 \mathrm{MeV}$. Figure 5 shows a slight mismatch between the mass energy absorption coefficients of PTFE and bone within the photon energy range of $0.1-0.2 \mathrm{MeV}$. However, the effect on dose distribution is expected to be negligible because the energy spectrum of $6 \mathrm{MV}$ X-rays, which are frequently used in IMRT, generally show less than a $2 \%$ contribution from the low energy region. Muscle, fat, and lung follow a certain trend in characteristics such as the mass attenuation coefficient and the mass energy absorption coefficients.

The LEGO-type multi-purpose dosimetry phantom was developed with the above-mentioned materials. The phantom's dosimetry conditions can be changed by stacking up the pieces. The air gap between the insertion holes and each heterogeneous material or dosimeter was minimized because the holes were carved to fit the dosimeters. Films can be sandwiched between the heterogeneous materials, and simultaneous measurements of vertical and lateral dose distributions are possible.

\subsection{Point-dose Measurement in Small Radiation Fields}

The developed phantom was used to measure the output factors for radiation fields ranging in size from $5 \times 5$ $\mathrm{cm}^{2}$ down to $0.5 \times 0.5 \mathrm{~cm}^{2}$ (see Figure 6). For the first test, 
Table 1. Physical Properties of Human Body Tissues and Selected Materials

\begin{tabular}{c|c|c|c|c|c}
\hline Body Tissues & $\rho\left[\mathrm{g} / \mathrm{cm}^{3}\right]$ & $Z_{\text {eff }}$ & Selected Materials & $\rho\left[\mathrm{g} / \mathrm{cm}^{3}\right]$ & $Z_{\text {eff }}$ \\
\hline Muscle & 1.04 & 7.71 & Polystyrene & 1.05 & 6.00 \\
\hline Fat & 0.95 & 6.63 & Polyethylene & 0.94 & 5.95 \\
\hline Bone & 1.92 & 11.18 & Polytetrafluoro-ethylene & 2 & 8.31 \\
\hline Lung & 0.26 & 7.80 & Polyurethane Foam & 0.28 & 6.94 \\
\hline
\end{tabular}
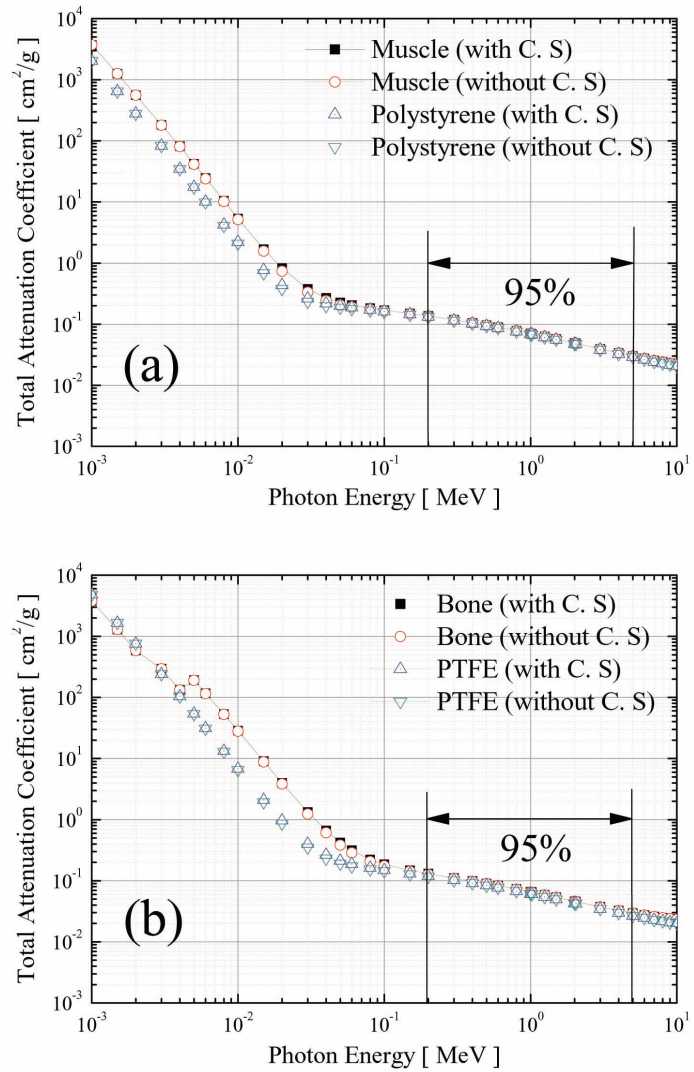

Fig. 4. Mass Attenuation Coefficients with or without Compton Scattering (C. S) for Body Tissues and Selected Phantom Materials: (a) Muscle and Polystyrene, (b) Bone and PTFE

we measured the dosimetry in a homogeneous phantom composed of only polystyrene. In this case, the ion chamber and the diode detector agreed within a $1 \%$ discrepancy down to a field size of $1.5 \times 1.5 \mathrm{~cm}^{2}$, while the differences between the two dosimeters were $11.7 \%$, $16.4 \%$, and $42.1 \%$ for the field sizes of $1 \times 1 \mathrm{~cm}^{2}, 0.7 \times 0.7$ $\mathrm{cm}^{2}$, and $0.5 \times 0.5 \mathrm{~cm}^{2}$, respectively. The output factors under the heterogeneous conditions were measured using a PTFE piece and an air cavity inserted into the polystyrene phantom. For the case of the PTFE insertion
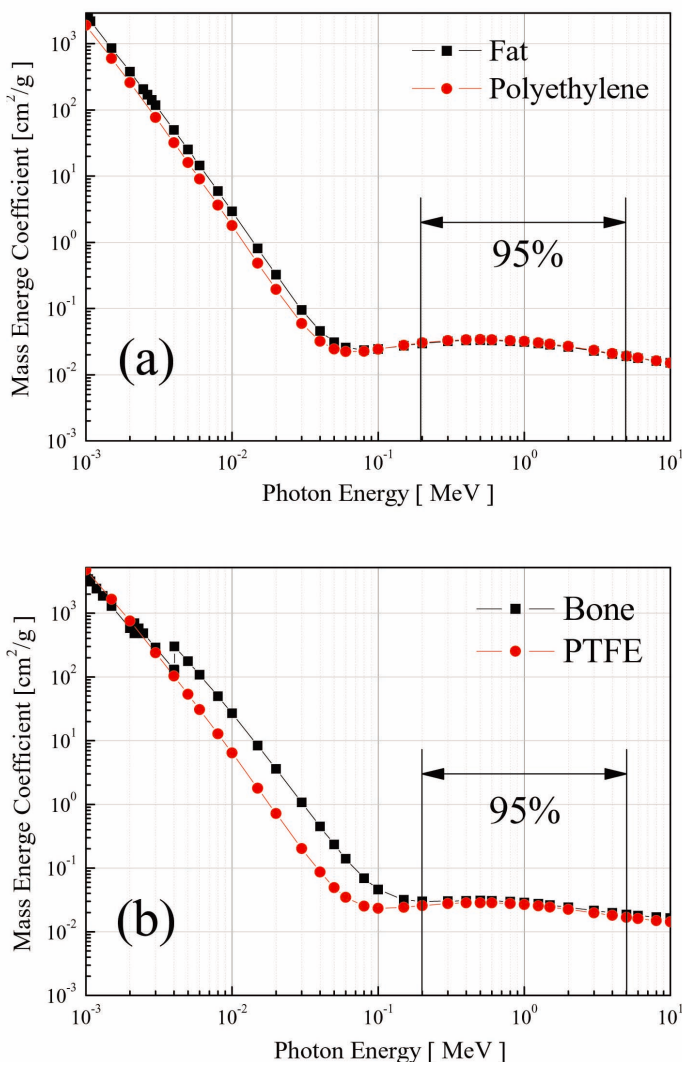

Fig. 5.Mass Energy Absorption Coefficients for Body Tissues and Selected Phantom Materials: (a) Fat and Polyethylene, and (b) Bone and PTFE

into the phantom, the results were similar to those in the homogeneous case. The ion chamber and the diode detector showed good agreement for the field sizes $5 \times 5$ $\mathrm{cm}^{2}-3 \times 3 \mathrm{~cm}^{2}$, and the differences measured for the three field sizes less than $1.5 \times 1.5 \mathrm{~cm}^{2}$ were $11.9 \%$, $23.3 \%$, and $39.9 \%$, respectively. When the air cavity was inserted into the polystyrene phantom, however, the measurements using the two types of dosimeter did not agree well for the field sizes less than $3 \times 3 \mathrm{~cm}^{2}$. The discrepancy was as large as $41 \%$ for the $0.7 \times 0.7 \mathrm{~cm}^{2}$ field.

The observed phenomena are due to a high dose- 


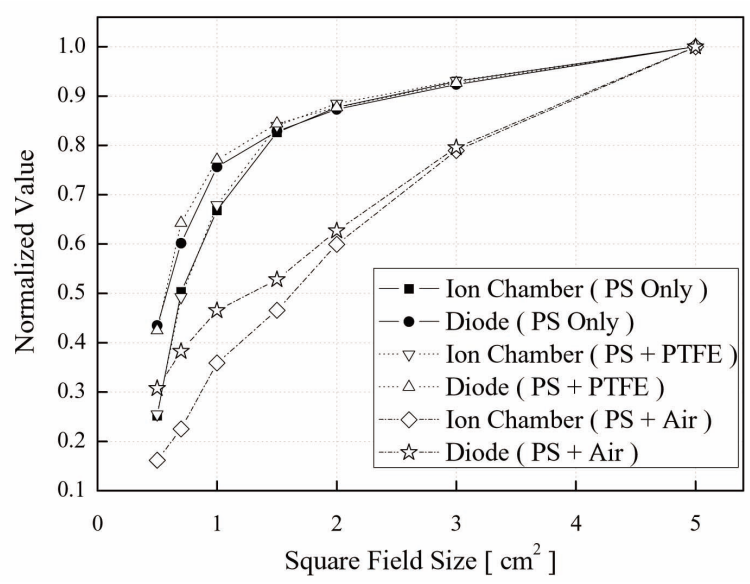

Fig. 6. Normalized Values of Point Doses Measured with Polystyrene only, PTFE, and Air Inserted into a Polystyrene Phantom

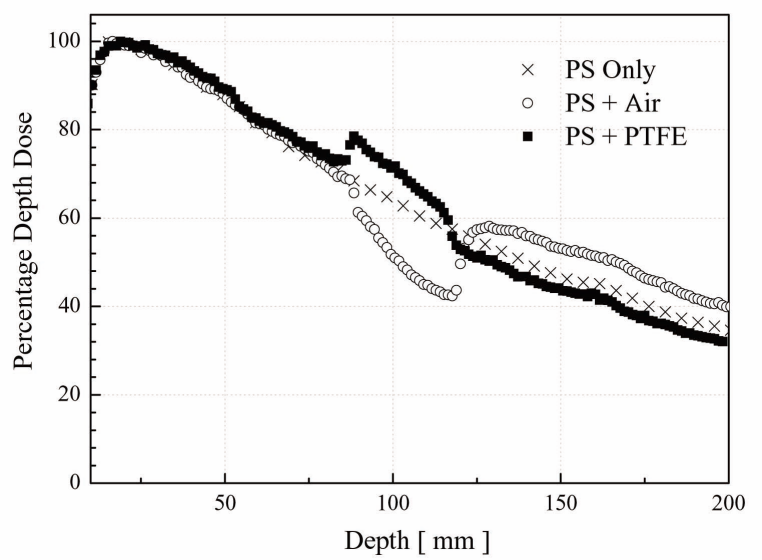

Fig. 7. Percent Depth Dose of $3 \times 3 \mathrm{~cm}^{2}$ Beam of $6 \mathrm{MV}-\mathrm{X}$ rays in a Phantom with $3 \times 3 \times 3 \mathrm{~cm}^{3}$ Air and PTFE Cubes at the Center of the Phantom

reported by Wilcox and Daskalov [6]. On the contrary, photons in the high density PTFE $\left(\rho_{\text {ptef }}=2 \mathrm{~g} / \mathrm{cm}^{3}\right)$ increase their interactions in the medium, thereby increasing the photon attenuation. Beyond the high density heterogeneous material, the relative radiological depth is larger and PDD is lower than that in a pure PS medium. The EBT film sandwiched in the bone substitute measured a higher dose since the stopping power ratio is larger in this environment [19].

\section{CONCLUSIONS}

In the present study, a LEGO-type dosimetry phantom was developed for diverse and convenient dosimetry for IMRT. In a test case study, we were able to verify that the phantom is useful for small-field dosimetry, even though the experimental results clearly showed that accurate measurement remains a challenge. Additionally, dose distributions across heterogeneous materials were measured using EBT films. Although our present study utilized tests with only simple beam geometries, more complicated combinations of beam sizes and heterogeneities are also possible when studying the measurements of point dose/dose distribution in heterogeneous media.

The data obtained herein can be compared with predictions from a radiation therapy treatment planning system to evaluate the accuracy of dose computation algorithms, or alternatively, the same data can be compared with theoretical predictions from Monte Carlo calculations for a more detailed understanding of the interface dynamics.

\section{ACKNOWLEDGMENTS}

This work was supported by the Nuclear R\&D Program under the auspices of the Korean government (MEST) (No. 20100018224, 20100023825). 


\section{REFERENCES}

[ 1 ] Y. Han, et al., "Dosimetry in an IMRT phantom designed for a remote monitoring program," Medical Physics, 35, 2519-2527 (2008).

[2] F. Sánchez-Doblado, et al., "Micro ionization chamber dosimetry in IMRT verification: Clinical implications of dosimetric errors in the PTV," Radiotherapy and Oncology 75, 342-348 (2005).

[ 3 ] R. Capote, F. Sánchez-Doblado, A. Leal, J. I. Lagares, and R. Arráns, "An EGSnrc Monte Carlo study of the microionization chamber for reference dosimetry of narrow irregular IMRT beamlets," Medical Physics, 31, 2416-2422 (2004).

[4] C. Y. Han, J. K. Kim, and K. S. Chung, "An Epithermal Neutron Beam Design for BNCT Using ${ }^{2} \mathrm{H}(\mathrm{d}, \mathrm{n})^{3} \mathrm{He}$ Reaction," Nucl. Eng. \& Tech., 31, 512-521 (1976).

[ 5 ] L. B. Leybovich, A. Sethi, and N. Dogan, "Comparison of ionization chambers of various volumes for IMRT absolute dose verification," Medical Physics, 30, 119-123 (2003).

[6] E. E. Wilcox and G. M. Daskalov, "Accuracy of dose measurements and calculations within and beyond heterogeneous tissues for $6 \mathrm{MV}$ photon fields smaller than $4 \mathrm{~cm}$ produced by Cyberknife," Medical Physics, 35, 2259-2266 (2008).

[ 7 ] H. S. Jeong, Y. Han, O Kum, and C. H. Kim, "Study on Small Field Dosimetry for High Energy Photon-based Radiation Therapy," Korean J Med Phys., 20, 290-297 (2009).

[8] J. W. Sohn, J. F. Dempsey, T. S. Suh, and D. A. Low, "Analysis of various beamlet sizes for IMRT with $6 \mathrm{MV}$ Photons," Medical Physics, 30, 2432-2439 (2003).

[9] I. J. Das, G. X. Ding, and A. Ahnesjö, "Small fields: Nonequilibrium radiation dosimetry," Medical Physics, 35, 206-215 (2008).
[10] H. S. Jeong, Y. Han, O Kum, and C. H. Kim, "Deconvolution of Ion Chamber Volume Effect for IMRT Absolute Dose Evaluation," The $5^{\text {th }}$ KOREA-JAPAN Joint Meeting on Medical Physics, Jeju, Korea, Sept. 10-12, (2008).

[11] C. Martens, C. D. Wagter, and W. D. Neve, "The Value of the PinPoint ion chamber for characterization of small field segments used in intensity-modulated radiotherapy," Physics in Medicine and Biology, 45 2519-2530 (2000).

[12] D. Radford, D. S. Followill, and W. F. Hanson, "Design of an Anthropomorphic Intensity Modulated Radiation Therapy Quality Assurance Phantom," The 22 $2^{\text {nd }}$ Annual EMBS International Conference, Chicago IL, USA, Jul. 23-28, (2000).

[13] C. Ma, et al., "A quality assurance phantom for IMRT dose verification," Physics in Medicine and Biology, 48, 561-572 (2003).

[14] C. Lee, and J. Lee., "Computational Anthropomorphic Phantoms for Radiation Protection Dosimetry: Evolution and Prospects," Nucl. Eng. \& Tech., 38, 239-250 (2006).

[15] Photon, Electron, Proton and Neutron Interaction Data for Body Tissues, ICRU report 46, International Commission on Radiation Units and Measurements, (1992).

[16] C. Shin, A Dual Energy Double Beam Method for Detection of Illicit Materials in $90^{\circ}$ Compton Scattering Inspection System, Doctoral Dissertation, Hanyang University, Seoul, Korea, (2007).

[17] National Institute of Standards and Technology (NIST), http://physics.nist.gov/PhysRefData/XrayMassCoef/cover. html

[18] F. H. Attix, Introduction to Radiological Physics and Radiation Dosimetry, 1st ed, Wiley-Interscience, New York, pp. 259-260, (1986).

[19] F. M. Khan, The physics of radiation therapy, 3rd ed, Lippincott Williams \& Wilkins, Philadelphia, pp. 254255, (2003). 